Published in final edited form as:

J Am Acad Child Adolesc Psychiatry. 2011 February ; 50(2): 108-118.e3. doi:10.1016/j.jaac.

2010.10.011.

\title{
Streptococcal upper respiratory tract infections and
}

exacerbations of tic and obsessive-compulsive symptoms: A

prospective longitudinal study

\author{
Dr. James F. Leckman, MD, Dr. Robert A. King, MD, Dr. Donald L. Gilbert, MD, Dr. Barbara \\ J. Coffey, MD, MS, Dr. Harvey S. Singer, MD, Dr. Leon S. Dure 4th, MD, Ms. Heidi Grantz, \\ MSW, Ms. Liliya Katsovich, MBA, Dr. Haiqun Lin, MD, PhD, Dr. Paul J. Lombroso, MD, Dr. \\ Ivana Kawikova, MD, PhD, Mr. Dwight R. Johnson, BS, Dr. Roger M. Kurlan, MD, and Dr. \\ Edward L. Kaplan, MD. \\ Drs. Leckman, King, Lombroso, and Kawikova, and Ms. Grantz and Ms. Katsovich are with the \\ Child Study Center and the Yale Center for Clinical Investigation, Yale University School of \\ Medicine. Dr. Gilbert is with the Division of Neurology at the Cincinnati Children's Hospital \\ Medical Center and the University of Cincinnati, School of Medicine. Dr. Coffey is with the Child \\ Study Center at New York University School of Medicine and the Nathan Kline Institute for \\ Psychiatric Research. Dr. Singer is with Johns Hopkins University School of Medicine. Dr. Dure is \\ with the Division of Child Neurology at the University of Alabama at Birmingham. Dr. Lin is with \\ Yale University School of Medicine. Mr. Johnson and Dr. Kaplan are with the University of \\ Minnesota Medical School. Dr. Kurlan is with the Atlantic Neuroscience Institute, Overlook \\ Hospital in Summit, NJ
}

\author{
Abstract \\ Objective-The objective of this blinded, prospective longitudinal study was to determine \\ whether new group A beta hemolytic streptococcal (GABHS) infections are temporally associated
}

\footnotetext{
Correspondence to Dr. James Leckman, Child Study Center, Yale University School of Medicine, 230 South Frontage Road, New Haven, CT 06520-7900, USA; james.leckman@yale.edu.

Disclosure: Dr. Leckman has received research support from the National Institutes of Health and the Tourette Syndrome Association. He has received salary support from the National Institutes of Health. He has received support from the Klingenstein Third Generation Foundation from the medical student fellowship program. He has received royalties from John Wiley and Sons, McGraw Hill, and Oxford University Press. Dr. Coffey receives research support from Boehringer Ingelheim, Bristol-Myers Squibb, Eli Lilly and Co., the National Institute of Mental Health, the National Institute of Neurological Disorders and Stroke, and the Tourette Syndrome Association. She has served on the advisory board for Jazz Pharmaceuticals, Eli Lilly and Co., Novartis, and the Tourette Syndrome Association. She has served on the speakers' bureau for the Tourette Syndrom Association. Dr. Singer has received research and salary support from the National Institutes of Health. He has received royalties from Elsevier, and has served as an editor for the journal Neurolograd. Dr. Gilbert has received honoraria from the American Academy of Pediatrics, the Tourette Syndrome Association/Center for Disease Control, the Movement Disorder Society, and the American Academy of Neurology. He has served on the advisory board for the Tourette Syndrome Association. He has received research support from the National Institutes of Health, Cincinnati Children's Hospital Research Foundation, the University of Cincinnati, and the Tourette Syndrome Association. He will receive salary support for clinical research from the Genzyme Corporation, Otsuka Pharmaceuticals, and Psyadon Pharmaceuticals, Inc. Dr. Kurlan has received research support from the National Institutes of Health, the Centers for Disease Control, the Michael J. Fox Foundation, Neauologix, Boehringer Ingelheim, and Kyowa. He has received salary support from the National Institutes of Health, Neurologix, Boehringer Ingelheim, and Kyowa. Dr. Lombroso has received research and salary support from the National Institute of Mental Health, the Institute for the Study of Aging, the American Health Assistant Foundation, and the Fragile X Foundation. Drs. King, Dure, Lin, Kawikova, and Kaplan, and Ms. Grantz, Mr. Johnson, and Ms. Katsovich report no biomedical financial interests or potential conflicts of interest.

Publisher's Disclaimer: This is a PDF file of an unedited manuscript that has been accepted for publication. As a service to our customers we are providing this early version of the manuscript. The manuscript will undergo copyediting, typesetting, and review of the resulting proof before it is published in its final citable form. Please note that during the production process errors may be discovered which could affect the content, and all legal disclaimers that apply to the journal pertain.
} 
with exacerbations of tic or obsessive-compulsive (OC) symptoms in children who met published criteria for Pediatric Autoimmune Neuropsychiatric Disorders Associated with Streptococcal infections (PANDAS). A group of children with Tourette syndrome and/or obsessive-compulsive disorder without a PANDAS history served as the (non-PANDAS) comparison group.

Method-Consecutive clinical ratings of tic and OC symptom severity were obtained for 31 PANDAS subjects and 53 non-PANDAS subjects. Clinical symptoms and laboratory values (throat cultures and streptococcal antibody titers) were evaluated at regular intervals during a 25 month period. Additional testing occurred at the time of any tic or OC symptom exacerbation. New GABHS infections were established by throat swab cultures and/or recent significant rise in streptococcal antibodies. Laboratory personnel were blinded to case or control status, clinical (exacerbation or not) condition, and clinical evaluators were blinded to the laboratory results.

Results-No group differences were observed in either the number of clinical exacerbations or the number of newly diagnosed GABHS infections. On only six occasions out of a total of 51 $(12 \%)$ a newly diagnosed GABHS infection was followed, within two months, by an exacerbation of tic and/or OC symptoms. In every instance, this association occurred in the non-PANDAS group.

Conclusions-This study provides no evidence for a temporal association between GABHS infections and tic/OC symptom exacerbations in children who meet the published PANDAS diagnostic criteria.

\section{Keywords}

obsessive-compulsive disorder; Tourette syndrome; group A beta hemolytic streptococci; streptococcal infections; pediatric neuropsychiatric disorders associated with streptococcal infections (PANDAS)

Tourette syndrome (TS) and pediatric-onset obsessive-compulsive disorder (OCD) are chronic, familial neuropsychiatric disorders that affect from 0.3 to $3 \%$ of the pediatric population. ${ }^{1,2}$ They are chronic disorders that are often associated with impairment and disability.

The etiologies of these disorders are unknown. It has been hypothesized that some individuals develop these disorders as a result of post-infectious autoimmune processes. ${ }^{3}$ As in Sydenham's chorea (SC), autoimmune responses following infections with group A beta hemolytic streptococci (GABHS) have been hypothesized to be responsible. ${ }^{3}$ Swedo et al. have identified this subgroup with the acronym PANDAS (Pediatric Autoimmune Neuropsychiatric Disorders Associated with Streptococcal infections). ${ }^{3}$ However, subsequent clinical and laboratory-based studies have failed to provide uniform support for this proposed subgroup. ${ }^{4-37}$

A major shortcoming of the PANDAS hypothesis has been the very small number of adequately designed prospective studies examining any temporal relationship between antecedent bona fide GABHS infections and the onset or exacerbations of tic and obsessivecompulsive (OC) symptoms. ${ }^{5-8}$ To determine whether there is such a specific relationship we conducted an intensive, blinded clinical and laboratory prospective cohort study that included PANDAS as well as non-PANDAS comparison subjects. We hypothesized that, if PANDAS is a unique clinical entity, then PANDAS cases would have more clinical exacerbations temporally linked to antecedent GABHS infections than non-PANDAS comparison subjects. The non-PANDAS subjects were diagnosed with either TS or pediatric-onset OCD and had no existing evidence of a prior association between GABHS infections and symptom onset or exacerbations as stated in the published criteria. ${ }^{3}$ 


\section{Method}

\section{Subjects}

All of the subjects were sequentially enrolled at one of six clinical settings (Yale University, Cincinnati Children's Hospital Medical Center, the New York University Child Study Center, Johns Hopkins University School of Medicine, the University of Alabama at Birmingham, and the University of Rochester School of Medicine) during a four and a half year period from July 2002 to December 2006. Approval by the Institutional Review Boards was obtained at all sites.

Case subjects met all five of the published diagnostic criteria for PANDAS described by Swedo et al. ${ }^{3}$ : (1) the presence of OCD and/or a chronic tic disorder (Tourette disorder, chronic motor or vocal tic disorder), defined as meeting lifetime Diagnostic and Statistical Manual of Mental Disorders, Fourth Edition (DSM-IV), diagnostic criteria; ${ }^{38}$ (2) age at onset between 3 years and the beginning of puberty; (3) clinical course characterized by the abrupt onset of symptoms or by a pattern of dramatic recurrent symptom exacerbations and remissions; (4) temporal relationship between GABHS infection and the clinical course of illness (onset and/or exacerbations) as reported by the subject or parent; and (5) at least one of the following symptoms appeared or became noticeably worse: motoric hyperactivity, choreiform movements, jerks of the hands, arms or legs, clumsiness, slurred speech, impaired dexterity or more difficulty drawing.

For criterion 4 above, we used a more exacting criterion. Specifically, we required documentation in the patient's medical record that there was a temporal relationship between an antecedent GABHS infection and the onset of the disorder as well as at least one exacerbation or that there was a temporal relationship between an antecedent GABHS infection and at least two exacerbations. We used time windows of 9 months for onset and 4 weeks for exacerbations. In addition, all PANDAS cases were required to be $\leq 16$ years of age. Informed consent had to be provided by a parent or guardian and assent provided by the subject. Exclusion criteria for "PANDAS cases" included the following: (1) Sydenham's chorea or a history of rheumatic fever; (2) presence of severe intellectual disability IQ<75, autism, or a secondary tic disorder other than PANDAS (e.g., drug induced or neuroacanthocytosis); (3) a psychiatric illness other than OCD or a tic disorder or ADHD that is the primary focus of treatment; (4) a major neurological disease (other than a tic disorder); (5) a CNS autoimmune disorder (e.g., multiple sclerosis, systemic lupus erythematosus, Behcet's) or a documented infection associated with neuropsychiatric symptoms (Lyme disease); (6) patient treated with corticosteroids, IVIG or plasma exchange during the prior 3 months; and/or (7) patient treated with antibiotics in the past 1 month.

Inclusion criteria for non-PANDAS comparison subjects were: (1) the presence of OCD and/or a chronic tic disorder by DSM-IV criteria; (2) age at onset between 3 and 14 years; (3) currently $\leq 16$ years of age; (4) following a review of the medical records and focused parental interviews, the clinical site director found no evidence that subject's clinical course of neuropsychiatric symptoms (onset or exacerbations) was temporally related to GABHS infections; and (5) informed consent provided by parent or guardian and assent provided by the subject. The same exclusion criteria for PANDAS case subjects were also applied to control subjects.

\section{Clinical assessments}

When a family entered the study, information concerning the patient was collected in a twostage process. ${ }^{5,7,8}$ Initially the families, in conjunction with experienced clinicians, completed the Yale Global Tic Severity Scale (YGTSS) ${ }^{39}$ ] and the Children's Yale-Brown Obsessive Compulsive Scale (CY-BOCS). ${ }^{40}$ Symptom severity in other domains was 
assessed using the DSM-IV Attention-Deficit/Hyperactivity Disorder (ADHD) Rating Scale, ${ }^{41}$ and the Child Global Assessment Scale for global functioning. ${ }^{42}$ The parent completed rating scales for ADHD (Conners' Abbreviated Symptom Questionnaire-Parent [ASQ-P]), ${ }^{43}$ and the child completed self-report ratings of depression (Child Depression Inventory-Short Version [CDI-SV] $)^{44}$ and anxiety (Multidimensional Anxiety Scale for Children [MASC]). ${ }^{45}$ The assessments for ADHD, depression, and anxiety symptoms were included to determine whether clinical exacerbations in association with GABHS infection are specific for tics and OCD or whether worsening is nonspecific and involves other childhood behavioral problems.

Clinical ratings were performed by assessors who were members of the existing clinical teams at each site with at least a masters-level degree. They were trained to reliability prior to the initiation of the study using videotaped assessments. Inter-rater reliability was maintained by periodic (every three to six months) co-rating among clinicians administering the C-YBOCS and YGTSS.

Comorbid psychiatric diagnoses, including ADHD, were made by three expert clinicians (JFL, RAK, and PJL) after reviewing all available information including data from the Schedule for Affective Disorders and Schizophrenia for School-Age Children. ${ }^{46}$ The same diagnostic panel used the PANDAS criteria to confirm cases as PANDAS or non-PANDAS. 3

\section{Longitudinal Evaluations}

Tic and OC symptom severities were assessed by an expert evaluator at the approximately monthly telephone contacts using the YGTSS and the CY-BOCS. Clinical evaluators were blinded to the results of streptococcal laboratory tests. However, they were not blinded with regard to the child's status as a PANDAS or non-PANDAS case. In-person visits occurred approximately every three months for monitoring of neuropsychiatric and collection of throat cultures for GABHS and blood samples for ASO and ADB titers. The parents were also asked to report any history of "sore throats", "colds", or other illnesses (especially those suggestive of streptococcal infections) that their child experienced during each three month interval. In addition, parents and/or subjects completed the self-report scales (ASQ-P; CDI$\mathrm{SV}$; and the MASC) every 4 weeks.

As previously described in detail, tic and/or OC symptom exacerbations were identified by using a two-stage algorithm. ${ }^{7,47}$ If an exacerbation occurred, the patient was immediately reevaluated and scheduled for two in-person visits when additional throat culture and serum specimens were collected (an "exacerbation" visit was scheduled as soon as possible and "follow-up" visit was scheduled eight weeks after the exacerbation visit). Most families completed their regularly scheduled encounters. The mean time between consecutive stafffamily encounters, across all patients, was 31.5 days (SD, 12.5). In six instances the time interval between visits exceeded 100 days.

\section{Throat cultures and serum specimens}

At the initial visit, duplicate throat swabs (collected simultaneously) and blood samples for ASO and ADB titers were obtained. All of the samples were sent by overnight courier to the Streptococcal Reference Laboratory at the University of Minnesota Medical School, Department of Pediatrics. In addition, parental authorization was obtained to contact each subject's pediatrician or primary care physician at the time of enrollment. These professionals then collected the monthly duplicate throat swabs that were obtained between the onsite study visits and sent the specimens directly to the Streptococcal Reference 
Laboratory. Laboratory personnel were blinded to case or control status and clinical status (i.e., clinical exacerbation visits).

Throat culture processing, streptococcal serogrouping, T-protein agglutination characterization, opacity factor determination, and $\mathrm{M}$ typing and/or $\mathrm{emm}$-gene sequence typing were performed in the streptococcus laboratory. ${ }^{48,49}$ Rapid antigen detection tests were not used. All of the $\beta$-hemolytic streptococci, not only group A, were serogrouped. The interval between throat swabs averaged 33.1 days (14.6) (range 3-156 days). Subsequently, duplicate throat swabs were obtained approximately monthly (mean days $=33.1$ [SD 14.6], range: $3-156)$ by either the primary care clinicians or the study investigators

Blood was collected in tubes with a clot activator and gel for serum separation (BD Biosciences, 367988). The samples were allowed to stand in room temperature for at least 30 min, after centrifugation serum was aliquoted and stored at $-80^{\circ} \mathrm{C}$. Tests for ASO and ADB antibodies were performed using the classical micro-titer plate methods with a dilution scheme based on $0.1 \log 10$ intervals. ${ }^{48}$ All serum samples from a given subject were tested in the same run to minimize effects of inter-test variation, and each run included appropriate "high" and "low" titer control sera. The average interval between serum specimens was approximately 13 weeks (88.7 days [SD 35.0], range: 3-156 days). Serum specimens were collected at each in-person visit (mean days $=88.7$ [SD 35.0], range: 17 to 290).

\section{GABHS infections}

In each instance, a designation of No, Possible, or a Definite new infection was made by DRJ and ELK based all available information concerning each subject and summarized in longitudinal graphs. ${ }^{49}$ The criteria used to identify a "Definite" new GABHS infection were the isolation of a GABHS strain with a corresponding $\log _{10}$ rise (of $\geq 0.20$ ) in either ASO and/or ADB antibody titers. For both the ASO and the ADB tests, an increase in titer of $\geq$ $0.2 \log _{10}$ between sequential specimens ( 13 week intervals) was considered to be significant. ${ }^{4}$ In order to satisfy the positive culture requirement, the prior culture had to be either negative or positive for a different strain of GABHS. The criteria used to identify a "Possible" new GABHS infection were either: 1) the isolation of a new GABHS strain without a corresponding rise in antibody titers; or 2) a temporally related $\log _{10}$ ASO or ADB titer increase of $\geq 0.20$.

If requested by the subject's primary care clinician, the results of the throat cultures were sent directly from the streptococcal laboratory by facsimile to his or her office.

Approximately two-thirds of the practitioners asked to receive this information. Any clinical decisions by the primary care physicians concerning the use of antibiotics were recorded for later analysis. This information was not shared with the study evaluators at the six clinical sites or at the laboratory.

\section{Medication status}

During the study, subjects were allowed to take their usual psychotropic medications for OCD, tics, or other conditions. Such treatment could be modified by the site investigator according to standard clinical practice. Consequently, each subject's medication history was monitored at each encounter. This information was collected from the parents, primary care physician, and the clinicians responsible for the treatment of the child's neuropsychiatric symptoms. Records were kept and coded concerning the use of antibiotics as well as specific classes of psychotropic agents (adrenergic agonists, neuroleptics, serotonin reuptake inhibitors and other antidepressants, mood stabilizers, psychostimulants, benzodiazepines, and other agents). Apart from antibiotics prescribed by pediatricians or other primary care 
clinicians, no immune-modifying or prophylactic and/or active antibiotic therapy specifically directed at PANDAS was given during the course of the study.

\section{Statistical analysis}

The baseline demographic and clinical data for cases and controls as well as PANDAS and non-PANDAS subjects were compared using either t-tests or Fisher Exact tests, as appropriate. Poisson regression model was used to compare rates of infections in the PANDAS and non-PANDAS groups.

The primary focus of this study was to determine the number of times a clinical exacerbation of tic and/or OC symptoms ${ }^{7}$ occurred in association with a newly diagnosed GABHS infection. Under the null hypothesis of no temporal association between infections and exacerbations, exacerbations were expected to be randomly distributed between the infection periods and the non-infection periods. For each subject, the expected number of "hits" (exacerbations that are temporally associated with documented GABHS infections) under the null hypothesis was calculated as the number of exacerbations experienced by the subject multiplied by the proportion of time that the subject spent in an infection period. This quantity was summed across subjects in each group of subjects (PANDAS or nonPANDAS cases) to obtain the total number of "hits" that would be expected by chance alone. Under the null hypothesis there would be no temporal association between infections and exacerbations.

Based on the results of earlier studies using similar methodologies, ${ }^{5,7}$ we selected two infection periods. First, we identified the exacerbations that occurred within 8 weeks after a newly diagnosed GABHS infection. We also included instances where the exacerbation occurred during the two week period prior to when the GABHS infection was first diagnosed (infection period $=10$ weeks). Second, based on the previous report by Kurlan et al., ${ }^{7}$ we also used a second infection period that extended from 2 weeks prior to five months following diagnosis of a new GABHS infection (infection period $=22$ weeks).

\section{RESULTS}

\section{Description of study cohort}

Apart from age, the PANDAS cases and the non-PANDAS comparison group had similar demographic and clinical characteristics (Table 1). As a consequence, all case vs. control comparisons included age as a covariate.

\section{Exacerbations}

The PANDAS cases and the non-PANDAS cases had very similar rates of tic and/or OC symptom exacerbations (PANDAS: 0.45 per person per year; non-PANDAS: 0.42 per person/year) (Table 2). The relative risk for PANDAS to Non-PANDAS after controlling for age was 1.27 with a 95\% confidence interval 0.75, 2.13 (non-significant). Of the 25 exacerbations identified in the PANDAS group only $6(24 \%)$ were associated with a simultaneous sudden increase $(\geq 25 \%)$ in anxiety, depression and/or ADHD symptoms. In the non-PANDAS group the rate was slightly lower at $18 \%$.

\section{GABHS infections}

The number of biological specimens collected is presented a supplemental table (See Table S1, available online). As presented in Table 3, the PANDAS cases and the non-PANDAS cases had very similar rates of newly diagnosed GABHS infections (Definite infection + Possible infection) (PANDAS: 0.36 per person per year; non-PANDAS: 0.39 per person/ 
year). Controlling for age, the relative risk for newly diagnosed GABHS infections was not increased in the PANDAS group relative to the non-PANDAS contrast group (Table 4).

\section{Antibiotic treatment}

Of the 52 Definite and Possible new GABHS infections, 21 episodes were treated by the primary care clinician with a variety of appropriate antibiotics. In the PANDAS group 60\% $(12 / 20)$ of the newly diagnosed GABHS infections were treated compared to $28 \%(9 / 32)$ in the non-PANDAS group. The proportion of treated GABHS infections among PANDAS subjects was significantly higher than among non-PANDAS subjects (Chi-square (1) $=5.2$, $p=0.02$ ).

\section{Hits}

Within the first infection period (10 weeks, see methods) only 6 hits $(10.1 \%)$ were observed out of a total of 59 clinical exacerbations of tics and/or OC symptoms. All of the hits occurred in non-PANDAS cases (Table 5). Remarkably, three of the six observed hits occurred in the same non-PANDAS case, an 11 year old boy diagnosed at entry with TS, OCD, ADHD and Generalized Anxiety Disorder. At his baseline (entry) assessment, no association between prior GABHS infections and tic worsening was documented. At each of these exacerbations his OC symptoms increased, but there was no sudden increase in his YGTSS, MASC, or CDI-SV scores.

Similar results were seen for the second infection period interval (22 weeks, see Methods) during which only 8 hits $(13.6 \%)$ were observed out of a total of 59 clinical exacerbations of tics and/or OC symptoms (see Table S2, available online).

In addition to considering the number of 'hits', we also examined the magnitude of the subsequent changes in tic and OC symptom severity following a new GABHS infection. This analysis also failed to detect significant differences between the PANDAS and nonPANDAS groups even when the patient's antibiotic treatment status was taken into account (see Supplement 1 and Table S3, available online).

\section{DISCUSSION}

In this prospective, blinded, case-control study of children who met published diagnostic criteria for PANDAS, there was no temporal association between clinical exacerbations and antecedent GABHS infections documented by microbiologic and/or immunologic criteria. The number of "hits" was higher in the non-PANDAS cases than in the PANDAS cases, although this difference was not statistically significant. Our findings support those from an earlier prospective, blinded, case-control study in which greater than $75 \%$ of clinical exacerbations were not temporally related to infections. However, in that study, the only "hits" occurred in the PANDAS cases, and PANDAS cases were significantly more likely to meet criteria for a new GABHS infection. ${ }^{7}$ The basis for this apparent difference is unlikely to be due to methodological differences, as each of six sites participating in this study were also sites for the earlier study ${ }^{7}$ and a virtually identical protocol was used in selecting and monitoring the PANDAS and non-PANDAS cases. Taken together, the implication from these two intensive, prospective studies (involving more than 160 unique subjects) is that there is no convincing evidence for an ongoing causal association between GABHS infections and tic/OC symptom exacerbations in children who meet the published PANDAS diagnostic criteria.

Given the binary nature of exacerbations (Yes or No), secondary analyses designed to determine if symptom severity worsening occurred following a newly diagnosed GABHS infection were also conducted. These analyses also failed to support a link between any 
documented GABHS infections and symptom exacerbations (See Supplement 1, available online). The one significant difference was that the PANDAS cases were significantly more likely to receive a course of antibiotics during episodes of pharyngitis from their primary care clinicians compared to the non-PANDAS cases. This may reflect the practice of many community-based practitioners to prescribe antibiotics once a diagnosis of PANDAS has been made.$^{50}$ On average, the use of these antibiotics did not influence subsequent changes in tic or OC symptom severity (see Table S3, available online).

Contrary to projections based on the retrospective study of Swedo et $\mathrm{al}^{3}{ }^{3}$ the PANDAS cases identified in this study experienced a lower rate of clinical exacerbations and documented bona fide new GABHS infections than expected. In addition, they did not typically show (during periods of tic or OC symptom exacerbation) a sudden increase in the severity of neuropsychiatric comorbidity including: emotional lability, intense anxiety, cognitive deficits, oppositional behaviors, motoric hyperactivity, choreiform movements, jerks of the hands, arms or legs, clumsiness, slurred speech, impaired dexterity or more difficulty drawing.

Another important potentially confounding issue in almost all of the earlier prospective longitudinal studies ${ }^{5,8}$ is that the criteria used to determine whether or not a new GABHS infection had occurred may have been flawed. In-depth analysis of prospectively and frequently collected culture and antibody data from this study, in conjunction with the earlier Kurlan et al. study ${ }^{7}$ has revealed that a single point or widely spaced cultures and/or only "elevated" ASO or ADB titers are often times misleading. ${ }^{49}$ Indeed, some cases had repeated positive throat cultures for the same strain of GABHS and their titers remained elevated over the entire 25 months of this study. ${ }^{49}$

These findings must be interpreted in light of several limitations of the study. ${ }^{37}$ First, although the expert clinical evaluators were blinded to the results of streptococcal laboratory tests and the decisions made by the patients' primary care clinicians to treat or not to treat GABHS infections, they were not blinded to the presumptive PANDAS status of the case. This lack of blindness could have influenced the clinical ratings leading to more or fewer exacerbations being detected.

Second, out of respect for the well being of the subjects in the study the primary healthcare providers were informed of the results of throat cultures. As a result, the patients' primary clinicians were free, if they chose to prescribe antibiotics for symptomatic or asymptomatic patients with positive cultures. This practice could have potentially limited the number of exacerbations observed. ${ }^{6}$

Third, we did not systematically monitor whether a child was referred for cognitive behavioral therapy (CBT) for either their tic or OC symptoms. This may have affected our results. ${ }^{51}$ However, only two of the sites were based in child psychiatry programs where tic and OC patients are routinely referred for CBT so that actual number of such cases is likely to have been relatively small.

Fourth, both the total number of clinical exacerbations and the total number of GABHS infections were lower than that had been estimated, raising the possibility that this study was underpowered.

Fifth, although the investigators in both this study and the earlier Kurlan et al. study ${ }^{7}$ prospectively identified PANDAS cases based on the published criteria, only a small minority of the clinical exacerbations recorded were consistent with the descriptions of PANDAS exacerbations in which the period of increased tic or OC symptom severity was associated with a sudden increase in the severity of psychiatric comorbidity. ${ }^{3}$ Consequently, 
if indeed PANDAS exists as a valid clinical entity, the diagnostic criteria may need to be strengthened. One set of proposed suggestions include (James Leckman, personal communication, July 2010): (1) eliminating cases where there was a sudden onset of a tic disorder in the absence of the sudden onset of OCD; (2) specifying that the acuity of symptom onset/exacerbation must be severe, dramatic, and proceeded from no/minimal symptoms to maximum severity within $24-48$ hours; and (3) requiring that the onset/ exacerbation of OC symptoms is accompanied by at least three (rather than just one) of the seven PANDAS associated symptoms (markedly increased level of anxiety; emotional lability, irritability, aggressive behavior; sudden difficulties with concentration or learning; developmental regression (loss of abilities); sleep disorder; sudden onset of motor dysfunction [dysgraphia, motoric hyperactivity, tics]; and/or urinary frequency or an increased urge to urinate) and that the acuity of the co-occurring symptoms must occur in the same sudden time interval as the OC symptoms.

Next, it is also worth noting that true PANDAS cases may be relatively rare so that it may be necessary to use a national referral base to recruit an adequate number of PANDAS cases as was true of the earlier studies conducted at NIH Clinical Center. ${ }^{3,27,28}$ In our view, future studies should focus in part on the new onset cases as this was not addressed in this study and it is a key element of the PANDAS hypothesis. However, if the focus is just on the treatment of new onset cases, this may leave in doubt whether PANDAS is chronic relapsing and remitting polyphasic illness if the intervention is efficacious. It will also be critical to exclude cases of SC.

Finally, although GABHS infections have been postulated as the main initial autoimmune response-inciting event contributing to the sudden onset of severe neuropsychiatric symptoms in a subgroup of patients, it is well documented that sudden OC and tic symptom onset or worsening can be triggered by other infectious agents (e.g. herpes simplex virus, varicella zoster virus, human immunodeficiency virus, Borrelia burgdorferi, Mycoplasma pneumoniae, as well as sinusitis, and the common cold). ${ }^{24,52-58}$ It will be important for clinicians and scientists to continue to work together across the disciplines of pediatrics, family medicine, neurology, child and adolescent psychiatry and immunology and microbiology to advance our knowledge and improve our understanding and care of these children regardless of the diagnostic label they carry.

This intensive study provides important additional and convincing evidence about the course of tic and OC symptoms in two groups of pediatric age subjects after these symptoms are established. During the prospective course of two years of intensive observation and care, no documentable differences emerged to suggest that GABHS infections were temporally related to either ongoing tic or OC symptoms or to exacerbations. The implication of this finding for patient care is that clinicians who see children who meet the published diagnostic criteria for PANDAS, ${ }^{3}$ particularly those whose exacerbations are limited to just tics or OC symptoms, typically do not need to perform throat cultures in the absence of symptoms of pharyngitis nor is there a convincing rationale for the use of prophylactic antibiotics. That said, further intensive longitudinal and treatment studies are warranted in younger children, at or close to the onset of their illness where there is clinical evidence of the abrupt onset or sudden worsening of OC and other neuropsychiatric symptoms.

\section{Supplementary Material}

Refer to Web version on PubMed Central for supplementary material. 


\section{Acknowledgments}

This research was funded in part by the Tourette Syndrome Association and the National Institutes of Health: R01MH061940 (JFL), K05 MH076273 (JFL), K02 MH01527 (PJL), M01RR006022, and RR00125.

The authors wish to thank the study families and participants for taking part in this study. We also wish to acknowledge Ms. Nancy Thompson, Ms. Virginia Eicher, and Barbara Peterson-Cremer, MD. Deborah Katz, and Matthew Monteiro at Child Study Center at Yale University; Tara D. Lipps, RN, CNP, with the Division of Neurology at the Cincinnati Children's Hospital Medical Center; Matthew Schrock, Tracey Rawls, MA, and Adeena Gabriel with the New York University Child Study Center; Dana Bridges, MS, with the Department of Neurology at the Johns Hopkins University School of Medicine; Donna Peneley, PN, with the Children's Hospital of Alabama; and Peter Como, PhD, and Pam Mapstone, MSN, PNP, with the Department of Neurology at the University of Rochester Medical Center for their valuable assistance in completing this study. We also wish to thank Cheryl Kunde and Amy Van Gheem, MD, with the World Health Organization Streptococcal Reference Laboratory and the Department of Pediatrics, University of Minnesota School of Medicine for their technical assistance in processing the cultures and in the antibody determinations.

\section{References}

1. Khalifa N, von Knorring A-L. Prevalence of the tic disorders and Tourette syndrome in a Swedish school population. Dev Med Child Neurol 2003;45:315-319. [PubMed: 12729145]

2. Geller DA. Obsessive-compulsive and spectrum disorders in children and adolescents. Psychiatr Clin North Am 2006;29(2):353-70. [PubMed: 16650713]

3. Swedo SE, Leonard HL, Garvey M, et al. Pediatric autoimmune neuropsychiatric disorders associated with streptococcal infections: clinical description of the first 50 cases. Am J Psychiatry 1998;155:264-271. [PubMed: 9464208]

4. Shulman ST. Pediatric autoimmune neuropsychiatric disorders associated with streptococci (PANDAS): update. Curr Opin Pediatr 2009;21(1):127-30. [PubMed: 19242249]

5. Luo F, Leckman JF, Katsovich L, et al. Prospective longitudinal study of children with tic disorders and/or obsessive-compulsive disorder: Relationship of symptom exacerbations to newly acquired streptococcal infections. Pediatrics 2004;113:e578-e585. [PubMed: 15173540]

6. Perrin EM, Murphy ML, Casey JR, et al. Does group A beta-hemolytic streptococcal infection increase risk for behavioral and neuropsychiatric symptoms in children? Arch Pediatr Adolesc Med 2004;158(9):848-56. [PubMed: 15351749]

7. Kurlan R, Johnson D, Kaplan EL. the Tourette Syndrome Study Group. Streptococcal infection and exacerbations of childhood tics and obsessive-compulsive symptoms: a prospective blinded cohort study. Pediatrics 2008;121(6):1188-97. [PubMed: 18519489]

8. Lin H, Williams KA, Katsovich L, et al. Streptococcal upper respiratory tract infections and psychosocial stress predict future tic and obsessive-compulsive symptom severity in children and adolescents with Tourette syndrome and/or obsessive-compulsive disorder. Biol Psychiatry 2010;67(7):684-91. [PubMed: 19833320]

9. Kirvan CA, Swedo SE, Heuser JS, Cunningham MW. Mimicry and autoantibody-mediated neuronal cell signaling in Sydenham chorea. Nat Med 2003;9:914-920. [PubMed: 12819778]

10. Church AJ, Dale RC, Lees AJ, et al. Tourette's syndrome: a cross-sectional study to examine the PANDAS hypothesis. J Neurol Neurosurg Psychiatry 2003;74(5):602-7. [PubMed: 12700302]

11. Pavone P, Bianchini R, Parano E, et al. Anti-brain antibodies in PANDAS versus uncomplicated streptococcal infection. Pediatr Neurol 2004;30(2):107-10. [PubMed: 14984902]

12. Singer HS, Hong JJ, Yoon DY, Williams PN. Serum autoantibodies do not differentiate PANDAS and Tourette syndrome from controls. Neurology 2005;65(11):1701-7. [PubMed: 16207842]

13. Kirvan CA, Swedo SE, Snider LA, Cunningham MW. Antibody-mediated neuronal cell signaling in behavior and movement disorders. J Neuroimmunol 2006;179:173-179. [PubMed: 16875742]

14. Dale RC, Candler PM, Church AJ, et al. Neuronal surface glycolytic enzymes are autoantigen targets in post-streptococcal autoimmune CNS disease. J Neuroimmunol 2006;172(1-2):187-97. [PubMed: 16356555]

15. Kansy JW, Katsovich L, McIver KS, et al. Identification of pyruvate kinase as an antigen associated with Tourette syndrome. J Neuroimmunol 2006;181:165-76. [PubMed: 17011640] 
16. Kirvan CA, Cox CJ, Swedo SE, Cunningham MW. Tubulin is a neuronal target of autoantibodies in Sydenham's chorea. J Immunol 2007;178:7412-7421. [PubMed: 17513792]

17. Cunningham MW, Kirvan C, Brimberg L, et al. Autoimmunity and behavior: Sydenham's chorea and related disorders. J Neuroimmunology 2008;203:185.

18. Singer HS, Gause C, Morris CM, et al. Serial immune markers do not correlate with clinical exacerbations in pediatric autoimmune neuropsychiatric disorders associated with streptococcal infections. Pediatrics 2008;121(6):1198-205. [PubMed: 18519490]

19. Gause C, Morris C, Vernekar S, et al. Antineuronal antibodies in OCD: comparisons in children with OCD-only, OCD+chronic tics and OCD+PANDAS. J Neuroimmunol 2009;214(1-2):118-24. [PubMed: 19628285]

20. Morris CM, Pardo-Villamizar C, Gause CD, Singer HS. Serum autoantibodies measured by immunofluorescence confirm a failure to differentiate PANDAS and Tourette syndrome from controls. J Neurol Sci 2009;276(1-2):45-8. [PubMed: 18823914]

21. Pollard, M.; Gause, CD.; Singer, et al. Serum antibody reactivity against tubulin is not altered in children with PANDAS and Tourette syndrome. Child Neurology Society; Louisville: 2009.

22. Martino D, Dale RC, Gilbert DL, et al. Immune activation and Tourette's syndrome. Mov Disord 2009;24(9):1267-79. [PubMed: 19353683]

23. Mell LK, Davis RL, Owens D. Association between streptococcal infection and obsessivecompulsive disorder, Tourette's syndrome, and tic disorder. Pediatrics 2005;116:56-60. [PubMed: 15995031]

24. Leslie DL, Kozma L, Martin A, et al. Neuropsychiatric disorders associated with streptococcal infection: A case-control study among privately insured children. J Am Acad Child Adolesc Psychiatry 2008;47(10):1166-72. [PubMed: 18724258]

25. Schrag A, Gilbert R, Giovannoni G, et al. Streptococcal infection, Tourette syndrome, and OCD. Neurology 2009;73:1256-1263. [PubMed: 19794128]

26. Gilbert DL, Kurlan RM. PANDAS: horse or zebra. Neurology 2009;73:1252-1253. [PubMed: 19794126]

27. Perlmutter SJ, Leitman SF, Garvey MA, et al. Therapeutic plasma exchange and intravenous immunoglobulin for obsessive-compulsive disorder and tic disorders in childhood. Lancet 1999;354(9185):1153-8. [PubMed: 10513708]

28. Giedd JN, Rapoport JL, Garvey MA, et al. MRI assessment of children with obsessive-compulsive disorder or tics associated with streptococcal infection. Am J Psychiatry 2000;157(2):281-3. [PubMed: 10671403]

29. Bombaci M, Grifantini R, Mora M, et al. Protein array profiling of tic patient sera reveals a broad range and enhanced immune response against Group A Streptococcus antigens. PLoS One 2009;4(7):e6332. [PubMed: 19623252]

30. Lit L, Gilbert DL, Walker W, Sharp FR. A subgroup of Tourette's patients over express specific natural killer cell genes in blood: a preliminary report. Am J Med Genet B Neuropsychiatr Genet 2007;144B(7):958-63. [PubMed: 17503477]

31. Lit L, Enstrom A, Sharp FR, Gilbert DL. Age-related gene expression in Tourette syndrome. J Psychiatr Res 2009;43(3):319-30. [PubMed: 18485367]

32. Leckman JF, Katsovich L, Kawikova I, et al. Increased serum levels of interleukin-12 and tumor necrosis factor-alpha in Tourette's syndrome. Biol Psychiatry 2005;57(6):667-673. [PubMed: 15780855]

33. Kawikova I, Leckman JF, Kronig H, et al. Decreased number of regulatory T cells suggests impaired immune tolerance in children with Tourette's syndrome. Biol Psychiatry 2007;61(3): 273-8. [PubMed: 16996487]

34. Kawikova I, Bart PX, Tobiasova Z, et al. Children with Tourette's syndrome may suffer IgA dysgammaglobulinemia: preliminary report. Biol Psychiatry 2010;67(7):679-83. [PubMed: 20006327]

35. Morer A, Chae W, Henegariu O, et al. Elevated expression of MCP-1, IL-2 and PTPR-N in basal ganglia of Tourette syndrome cases. Brain Behav Immun 2010;24(7):1069-1073. [PubMed: 20193755] 
36. Yaddanapudi K, Hornig M, Serge R, et al. Passive transfer of streptococcus-induced antibodies reproduces behavioral disturbances in a mouse model of pediatric autoimmune neuropsychiatric disorders associated with streptococcal infection. Mol Psychiatry 2010;15(7):712-26. [PubMed: 19668249]

37. Murphy TK, Kurlan R, Leckman J. The immunobiology of Tourette's disorder, pediatric autoimmune neuropsychiatric disorders associated with Streptococcus, and related disorders: a way forward. J Child Adolesc Psychopharmacol 2010;20(4):317-31. [PubMed: 20807070]

38. American Psychiatric Association. Diagnostic and Statistical Manual of Mental Disorders. 4. Washington, DC: American Psychiatric Association; 1994. p. 393-444.(DSM-IV)

39. Leckman JF, Riddle MA, Hardin MT, et al. The Yale Global Tic Severity Scale: Initial testing of a clinician-rated scale of tic severity. Am J Child Adolesc Psychiatry 1989;28:566-573.

40. Scahill L, Riddle MA, McSwiggan-Hardin MT, et al. Children's Yale-Brown Obsessive Compulsive Scale: Reliability and validity. Am J Child Adolesc Psychiatry 1997;36:844-852.

41. DuPaul GJ. Parent and teacher ratings of ADHD symptoms: psychometric properties in a community-based sample. J Clin Child Psychol 1991;20 (3):245-253.

42. Shaffer D, Gould MS, Brasic J. A children's global assessment scale (CGAS). Arch Gen Psychiatry 1983;40 (11):1228-1231. [PubMed: 6639293]

43. Goyette CH, Conners CK, Ulrich RF. Normative data on Revised Conners' Parent and Teacher Rating Scales. J Abn Child Psychol 1978;6 (2):221-236.

44. Kovacs, M. The Children's Depression Inventory (CDI). Los Angeles, CA: Western Psychological Services; 1992.

45. Stallings, P.; March, JS. Assessment. In: March, JS., editor. Anxiety Disorders in Children and Adolescents. New York, NY: Guilford Press; 1995. p. 125-147.

46. Kaufman J, Birmaher B, Brent D, et al. Schedule for Affective Disorders and schizophrenia for school-age children present and lifetime version: Initial reliability and validity data. Am J Child Adolesc Psychiatry 1997;36:980-988.

47. Lin H, Yeh CB, Peterson BS, Scahill L, et al. Assessment of symptom exacerbations in a longitudinal study of children with Tourette's syndrome or obsessive-compulsive disorder. J Am Acad Child Adolesc Psychiatry 2002;41(9):1070-7. [PubMed: 12218428]

48. Johnson, DR.; Kaplan, EL.; Smramek, J., et al. Laboratory Diagnosis of Group A Streptococcal Infections. Geneva, Switzerland: World Health Organization; 1996.

49. Johnson DR, Kurlan R, Leckman JF, Kaplan EL. The human immune response to streptococcal extracellular antigens: Clinical, diagnostic and potential pathogenetic implications. Clin Infect Dis 2010;50:481-490. [PubMed: 20067422]

50. Gabbay V, Coffey BJ, Babb JS, et al. Pediatric autoimmune neuropsychiatric disorders associated with streptococcus: comparison of diagnosis and treatment in the community and at a specialty clinic. Pediatrics 2008;122(2):273-278. [PubMed: 18676543]

51. Storch EA, Murphy TK, Geffken GR, Mann G, Adkins J, Merlo LJ, Duke D, Munson M, Swaine Z, Goodman WK. Cognitive-behavioral therapy for PANDAS-related obsessive-compulsive disorder: findings from a preliminary waitlist controlled open trial. J Am Acad Child Adolesc Psychiatry 2006;45(10):1171-1178. [PubMed: 17003662]

52. Jankovic, J.; Kwak, C. Tics in other neurological disorders. In: Kurlan, R., editor. Handbook of Tourette's syndrome and related tic and behavioural disorders. New York: Marcel Dekker; 2004

53. Dale RC, Church AJ, Heyman I. Striatal encephalitis after Varicella zoster infection complicated by tourettism. Mov Disord 2003;18:1554-1556. [PubMed: 14673900]

54. Cardoso, F. Infectious and transmissible movement disorders. In: Jankovic, J.; Tolosa, E., editors. Parkinson's disease and movement disorders. 4. Baltimore: Williams and Wilkins; 2002. p. 930-940.

55. Müller N, Riedel M, Blendinger C, et al. Mycoplasma pneumoniae infection and Tourette's syndrome. Psychiatry Res 2004;129:119-125. [PubMed: 15590039]

56. Hoekstra PJ, Manson WL, Steenhuis MP, et al. Association of common cold with exacerbations in pediatric but not adult patients with tic disorder: a prospective longitudinal study. J Child Adolesc Psychopharm 2005;15:285-292. 
57. Termine C, Uggetti C, Veggiotti P, et al. Long-term follow-up of an adolescent who had bilateral striatal necrosis secondary to Mycoplasma pneumoniae infection. Brain Dev 2005;27:62-65. [PubMed: 15626544]

58. Ercan TE, Ercan G, Severge B, et al. Mycoplasma pneumoniae infection and obsessive-compulsive disease: a case report. J Child Neurol 2008;23:338-340. [PubMed: 18079308] 


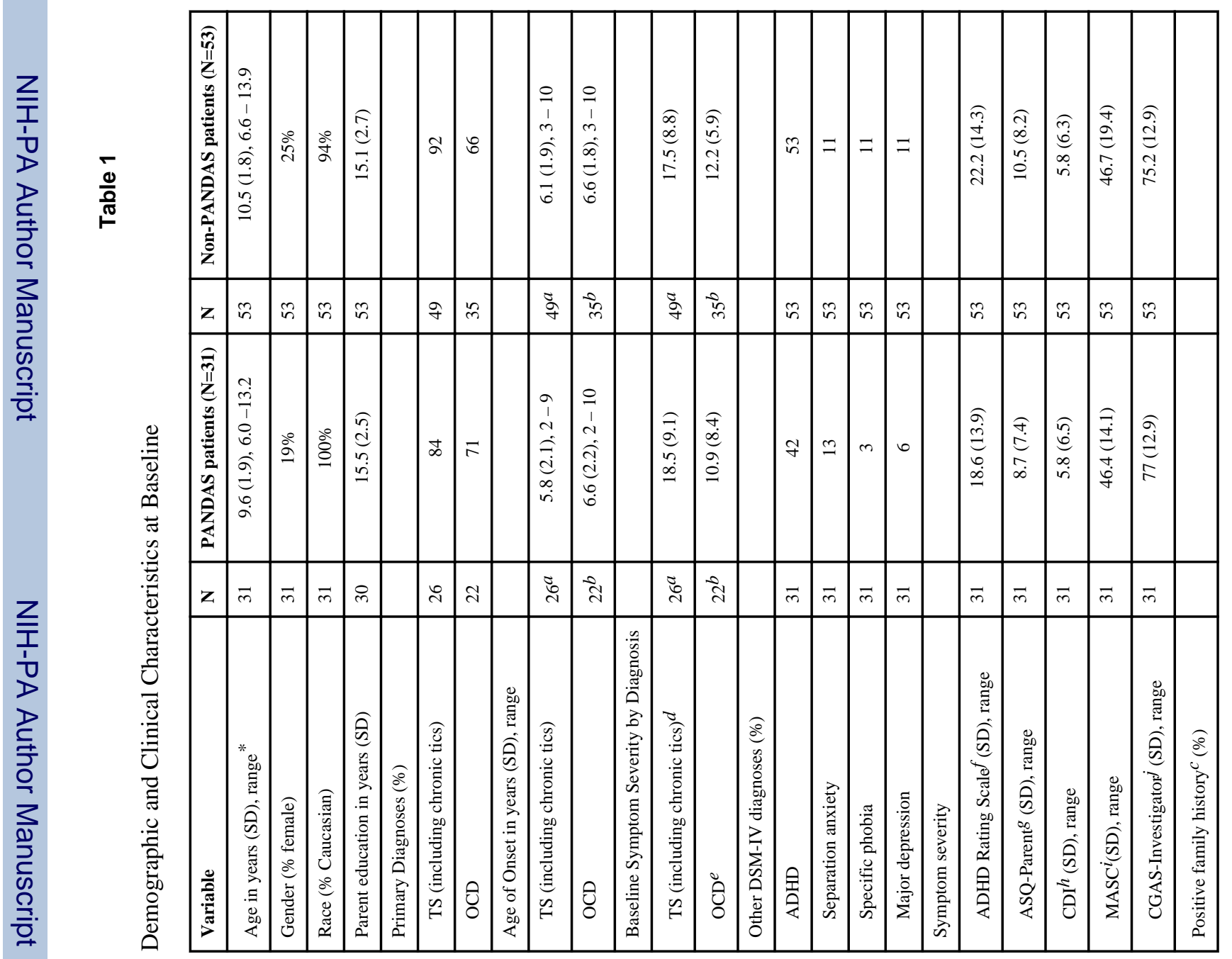




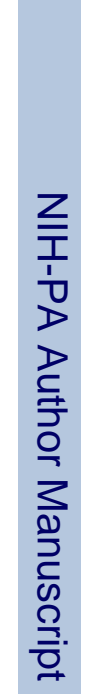

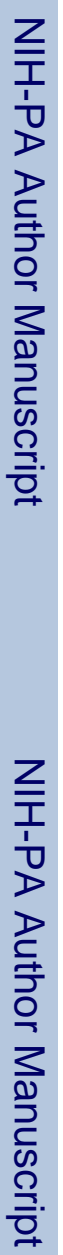

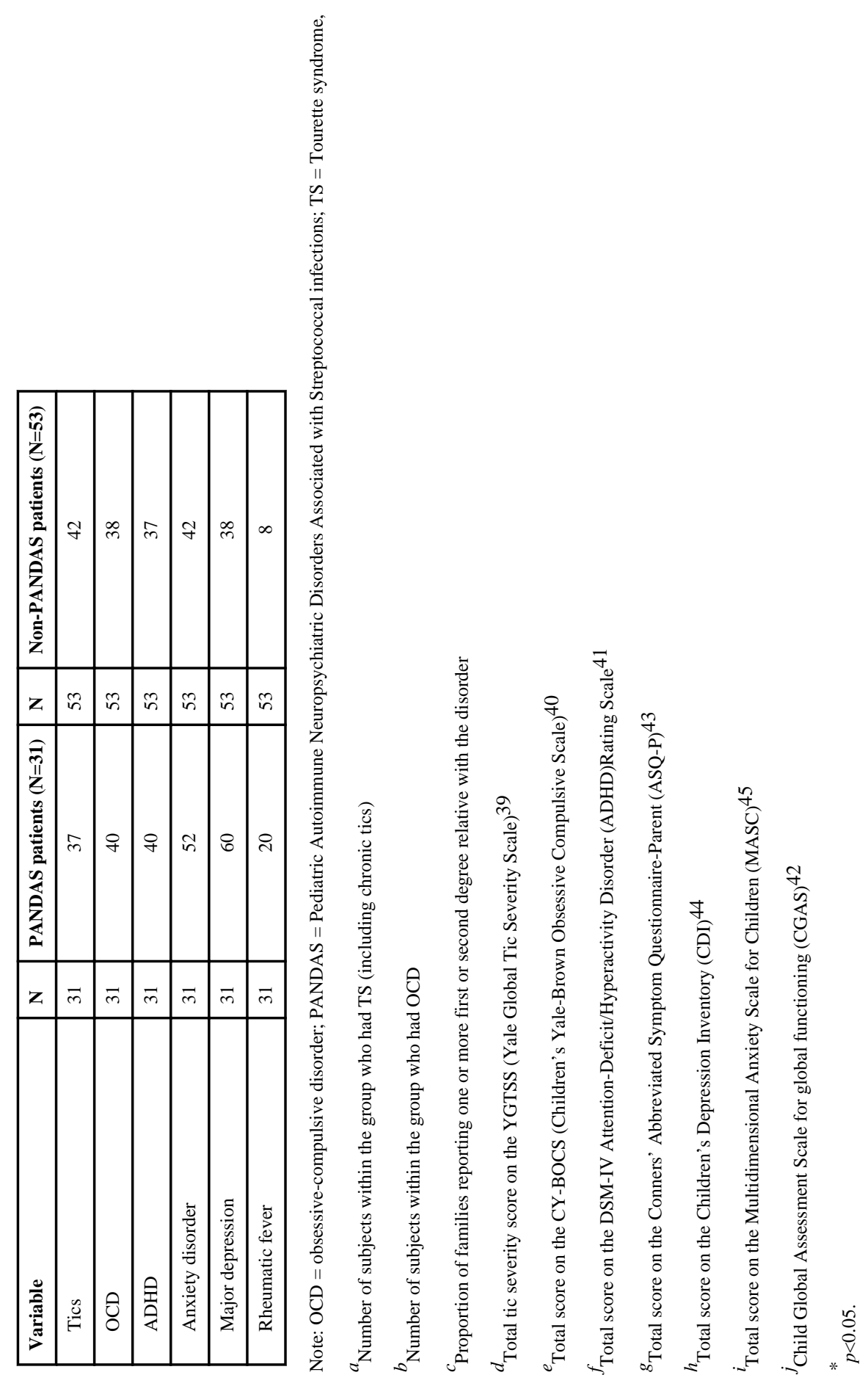


Table 3

Newly Diagnosed Group A Beta Hemolytic Streptococcal infections

\begin{tabular}{|l|c|c|c|c|}
\hline Group & $\begin{array}{c}\text { Period of } \\
\text { observation } \\
\text { (months) }\end{array}$ & $\begin{array}{c}\text { Newly diagnosed } \\
\text { definite GABHS } \\
\text { infections (infections } \\
\text { per person per year) }\end{array}$ & $\begin{array}{c}\text { Newly diagnosed } \\
\text { possible GABHS } \\
\text { infections (infections per } \\
\text { person per year) }\end{array}$ & $\begin{array}{c}\text { Newly diagnosed definite } \\
\text { + possible GABHS } \\
\text { infections (infections per } \\
\text { person per year) }\end{array}$ \\
\hline All patients (N=84) & 1646.4 & $26(0.19)$ & $26(0.19)$ & $52(0.38)$ \\
\hline PANDAS patients (N=31) & 663.1 & $7(0.13)$ & $13(0.23)$ & $20(0.36)$ \\
\hline Non-PANDAS patients (N=53) & 983.3 & $19(0.23)$ & $13(0.16)$ & $32(0.39)$ \\
\hline
\end{tabular}

Note: No significant differences were observed comparing the rates of infections. GABHS = group A beta hemolytic streptococci; PANDAS = pediatric autoimmune disorders associated with streptococcal infections. 


\section{Table 4}

Relative Risks for Newly Diagnosed Group A Beta Hemolytic Streptococcal infections controlling for age

\begin{tabular}{|l|c|c|}
\hline PANDAS/Non-PANDAS & Relative Risk PANDAS/Non-PANDAS & 95\% CI \\
\hline Relative Risk for definite GABHS Infections & 0.50 & $0.20,1.22$ \\
\hline Relative Risk for possible GABHS Infections & 1.19 & $0.54,2.65$ \\
\hline Relative Risk for definite + possible GABHS Infections & 0.81 & $0.45,1.43$ \\
\hline
\end{tabular}

Note: None of the Relative Risks above were statistically significant. $\mathrm{CI}=$ Confidence Interval; GABHS = group A beta hemolytic streptococci; PANDAS = pediatric autoimmune disorders associated with streptococcal infections. 


\section{Table 5}

Observed vs. expected number of hits when a newly diagnosed group A beta hemolytic streptococcal infections occurred in a 10 week time window surrounding to a tic and/or osbsessive-compulsive symptom exacerbation $^{a}$

\begin{tabular}{|c|c|c|c|}
\hline Infection classification $b$ & Number of hits & Expected number of hits by chance & $95 \% \mathrm{CI}$ \\
\hline \multicolumn{4}{|l|}{ All subjects } \\
\hline Definite GABHS infections & $3^{c}$ & 2.3 & $0.62,8.77$ \\
\hline Possible GABHS infections & $3^{c}$ & 1.5 & $0.62,8.77$ \\
\hline Definite or Possible GABHS infections & $6^{c}$ & 3.8 & $2.2,13.1$ \\
\hline \multicolumn{4}{|l|}{ PANDAS only } \\
\hline Definite GABHS infections & - & 0.5 & - \\
\hline Possible GABHS infections & - & 0.5 & - \\
\hline Definite or Possible GABHS infections & - & 1.0 & - \\
\hline \multicolumn{4}{|l|}{ Non-PANDAS } \\
\hline Definite GABHS infections & $3^{c}$ & 1.8 & $0.62,8.77$ \\
\hline Possible GABHS infections & $3^{c}$ & 1.0 & $0.62,8.77$ \\
\hline Definite or Possible GABHS infections & $6^{c}$ & 2.8 & $2.2,13.1$ \\
\hline
\end{tabular}

Note: $\mathrm{CI}=$ confidence interval; GABHS = group A beta hemolytic streptococcal infection; OC = obsessive-compulsive; PANDAS = Pediatric Autoimmune Disorder Associated with Streptococcal infections

$a_{\text {Hits }}=$ For each subject, the expected number of "hits" under the null hypothesis was calculated as the number of exacerbations experienced by the subject multiplied by the proportion of time that the subject spent two weeks prior to the detection of a new GABHS infection plus eight weeks following the detection of a new GABHS infection. This quantity was summed across subjects in each group of subjects (PANDAS or control) to obtain the total number of "hits" that would be expected by chance alone. Please see text for the rationale for including the two week interval before a new GABHS infection was diagnosed. Table S2, available online, presents a similar analysis for a second infection interval defined as two weeks prior to the detection of a new GABHS infection plus 20 weeks following the detection of a new GABHS infection. This longer interval was considered in an exploratory analysis because of the findings reported by Kurlan et al. ${ }^{7}$

$b_{\text {See text }}$

${ }^{c}$ In one non-PANDAS subject, two exacerbations occurred within 2 months following definite GABHS infection and one exacerbation following a possible GABHS infection. 